\title{
Reduced Expression Level of GPX2 in T1 Bladder Cancer and its Role in Early-phase Invasion of Bladder Cancer
}

\author{
AKINORI MINATO ${ }^{1}$, HIROTSUGU NOGUCHI $^{2}$, REI OHNISHI ${ }^{1}$, \\ IKKO TOMISAKI $^{1}$, TOSHIYUKI NAKAYAMA ${ }^{3}$ and NAOHIRO FUJIMOTO ${ }^{1}$
}

${ }^{1}$ Department of Urology, School of Medicine, University of Occupational and Environmental Health, Kitakyushu, Japan; ${ }^{2}$ Department of Pathology, Field of Oncology Graduate School of Medical and Dental Science, Kagoshima, Japan;

${ }^{3}$ Department of Pathology, School of Medicine, University of Occupational and Environmental Health, Kitakyushu, Japan

\begin{abstract}
Background/Aim: The role of glutathione peroxidase 2 (GPX2) expression in urothelial carcinoma (UC) is rarely reported. The aim of this study was to assess the expression status of GPX2 in UC of the bladder. Materials and Methods: We collected samples from 112 patients treated with radical cystectomy for immunohistochemical study. Results: Following immunohistochemical analysis of the specimens, 86 (76.8\%) had weak GPX2 expression. In cases with consistent GPX2 expression within the same lesion, the levels of GPX2 showed significant decreases from pTa to pT1 (47.1\%) compared to those from pT1 to pT2 (5.9\%) (p=0.017). Specimens obtained with transurethral resection before cancer progressed to muscle invasive bladder cancer showed that pT1 had a lower expression for GPX2 than that of pTa $(63.3 \% \mathrm{vs.}$ 93.3\%; $p=0.009)$. Conclusion: The decrease in GPX2 expression among those with UC of the bladder may be involved in the early step of cancer invasion.
\end{abstract}

Bladder cancer is the second most common urological malignancy after prostate cancer (1). Urothelial carcinoma (UC) is the predominant histology of bladder cancer; however, the molecular mechanisms of UC carcinogenesis are varied and not well-understood (2). There is some evidence suggesting that oxidative stress and bladder carcinogenesis are closely related $(3,4)$; the activity of

This article is freely accessible online.

Correspondence to: Akinori Minato, MD, Ph.D., Department of Urology, School of Medicine, University of Occupational and Environmental Health, 1-1, Iseigaoka, Yahatanishi-ku, Kitakyushu, 807-8555, Japan. Tel: +81 936917446, Fax: +81 936038724, e-mail: a-minato@med.uoeh-u.ac.jp

Key Words: Urothelial carcinoma, bladder cancer, glutathione peroxidase 2 (GPX2), immunohistochemistry, invasion. antioxidant enzymes with a defence mechanism role against oxidative toxicity has been observed in various cancer cells $(5,6)$. Glutathione peroxidase 2 (GPX2) is a selenoprotein that belongs to the antioxidant enzyme glutathione peroxidase family (GPX1-8) (5). It was first identified in the gastrointestinal system where it likely acts as a barrier against hydroperoxide absorption (7). In addition, the expression of GPX2 has been detected in several types of cancer cells (8-13) and was found to be a prognostic marker candidate in castration-resistant prostate cancer (14). However, the role of GPX2 expression in UC of the bladder is rarely reported, and yet to be fully elucidated. The aim of this study was to assess the clinicopathologic value of GPX2 as it relates to $\mathrm{UC}$ of the bladder.

\section{Materials and Methods}

Patients and tissue specimens. All the intended procedures in the present study, including the use of specimens from human subjects were approved by the Institutional Review Board of the University of Occupational and Environmental Health (UOEH) with the approval number H28-047. We conducted a retrospective review of 139 consecutive patients with muscle invasive bladder cancer (MIBC) without distant metastasis who had undergone radical cystectomy (RC), pelvic lymphadenectomy, and urinary diversion at the UOEH Hospital (Kitakyushu, Japan) between March 2003 and March 2017. We collected samples from 112 patients who had histologically confirmed UC including mixed variant histology for immunohistochemical study and survival analysis. Patients with non-UC such as squamous cell carcinoma and adenocarcinoma and those with no residual tumors (pT0) or carcinoma in situ only, were excluded from the present study.

Formalin-fixed, paraffin-embedded tissue blocks were provided by our Department of Pathology. Two pathologists examined all transurethral resections of bladder tumour (TURBT) tissue and RC specimens to confirm the histopathologic features based on 2016 World Health Organization/International Society of Tumors of the Urinary system and Male Genital Organs classification (15). These tissues were stained with hematoxylin and eosin, and immunohistochemistry (IHC) was performed on preparations of sequential sections. 

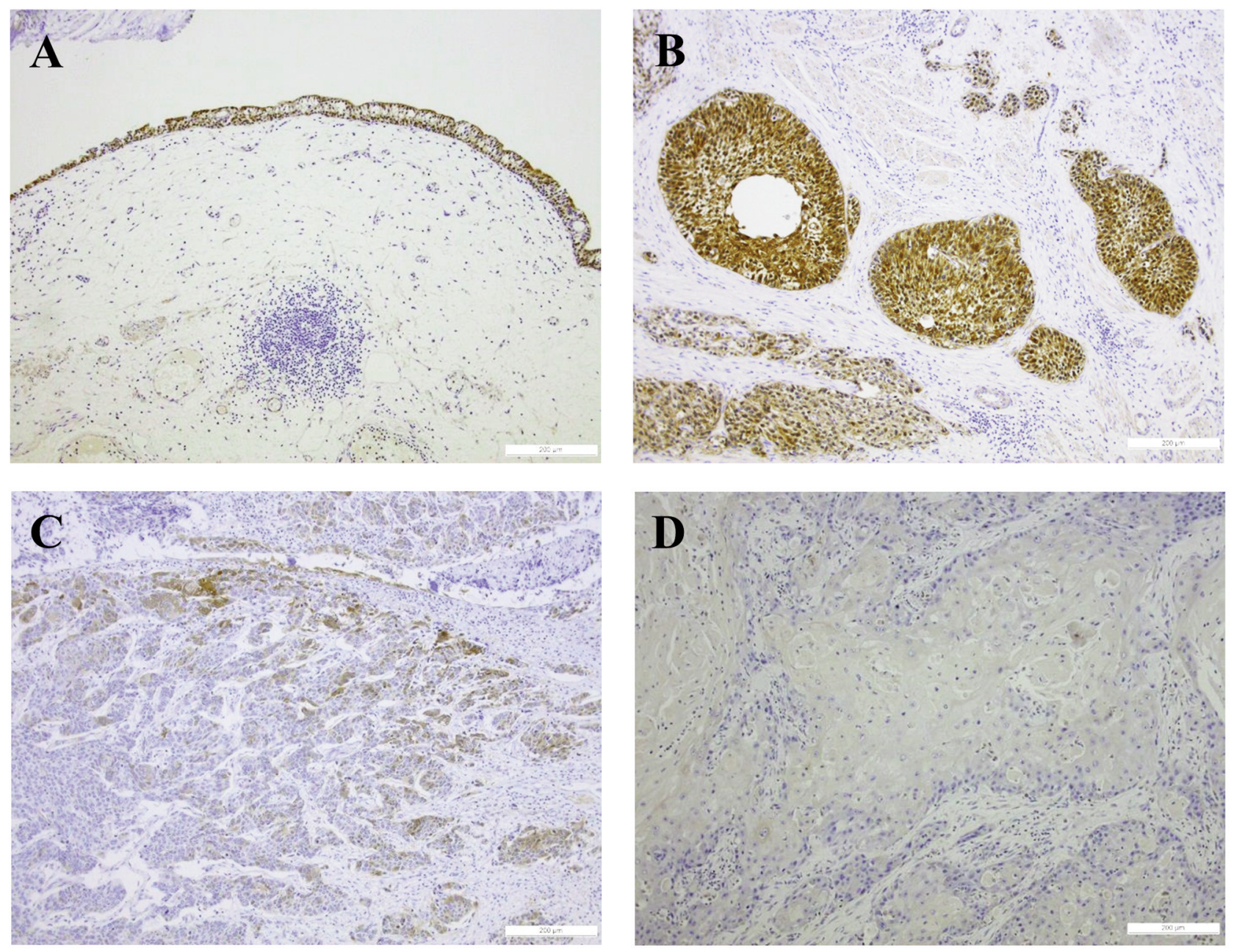

Figure 1. Representative immunohistochemistry for GPX2 staining in normal urothelial cells (A) (3+) and tumor cells $(B, C, D)(3+, 2+, 1+)$ of the bladder.

Immunohistochemical staining of GPX2. IHC was performed according to the antibody-linked dextran polymer method for antibody-bridge labelling, with hematoxylin counterstaining (EnVision; DAKO). Deparaffinized and rehydrated $4 \mu \mathrm{m}$ sections were incubated in $10 \% \mathrm{H}_{2} \mathrm{O}_{2}$ for 5 min to block the endogenous peroxidase activity. The sections were subsequently rinsed and incubated with a primary antibody targeting GPX2 (rabbit polyclonal, Atlas Antibodies, Stockholm, Sweden) at a dilution of 1:50 for $60 \mathrm{~min}$. The second antibody-peroxidase-linked polymers were then applied. After counterstaining with Meyer's hematoxylin, the sections were observed under a light microscope. Each section was first scanned at low power for all fields (original magnification: $\times 40$ ) using tumor and non-tumor tissues, respectively, in order to determine the heterogeneity of distribution. The number of positive cells showing cytoplasmic staining was recorded at the site of invasion. For IHC of GPX2, we used normal urothelial cells as the positive control. Incubation without the primary antibody was used as a negative control. Information on necrotic tissue, stromal cells, and lymphoid cells was not recorded.
The degree of immunoreactivity for GPX2 was assessed in each case semi-quantitatively by evaluating the proportion of positive cells relative to the total number of UC cells. We selected and validated immunohistochemical cut-off scores for GPX2 positivity, based on the findings of a receiver operating characteristic curve analysis. Finally, all patients were divided into two groups, as follows: those with normal findings, GPX2 equal to or more than $60 \%(3+)$; and those with weak (weakly positive) findings, less than $60 \%(2+, 1+$, and 0$)$. All histological and immunohistochemical slides were evaluated by two independent pathologists who were blinded to the clinicopathologic data.

Prognostic assessment. All patients underwent RC. Postoperative follow-up examinations comprised of physical examinations, laboratory tests, and CT scans, which were conducted every six months until the fifth year and annually thereafter. When symptoms appeared, the appropriate additional examinations were conducted. The overall survival (OS) duration was calculated from the date the $\mathrm{RC}$ was performed to the date of death, due to any cause or to the 
date of the last follow-up, if the patient was alive. The disease-free survival (DFS) duration was calculated from the date the RC was performed to the date of the first clinical recurrence or the date of death due to any cause or the last follow-up, if the patient had no known recurrence.

Statistical analysis. All statistical analyses were performed using EZR (Easy R, Vienna, Austria), which is a graphical user interface for R (The R Foundation for Statistical Computing). Fisher's exact test and Kruskal-Wallis test were used to examine the associations between categorical variables. Continuous variables were divided into two groups according to their median values. The MannWhitney $U$-test compared the continuous variables. OS and DFS were estimated using the Kaplan-Meier method and the log-rank test. $p \leq 0.05$ was considered statistically significant.

\section{Results}

IHC of GPX2 expression. GPX2 expression was strongly detectable (3+) in normal urothelial cells, whereas GPX2 expression was lower in tumor cells (Figure 1). Of 112 specimens, $26(23.2 \%)$ revealed normal GPX2 expression (3+) while 37,37 , and $12(33.0 \%, 33.0 \%$ and $10.7 \%$, revealed $2+, 1+$, and 0 , or weak expression, respectively.

Relationship between GPX2 expression and clinicopathological characteristics. According to the IHC results indicating GPX2 in tumor cells, we divided the patients into two groups: the GPX2-normal (3+) group and GPX2-weak $(2+, 1+$, and 0) group. There were no substantial differences in clinicopathologic characteristics between the groups (Table I). The GPX2-weak group included a larger proportion of patients with UC who had variants in histology, but the difference was not significant $(44.2 \%$ vs. $23.1 \%$; $p=0.068)$.

Survival analysis for GPX2 expression. The median followup time was 32 months (range=4-143 months), during which $50(44.6 \%)$ patients experienced recurrence and $43(38.4 \%)$ died. The 5-year OS rate of the GPX2-normal group compared to the GPX2-weak groups was $57.2 \%$ vs. $58.8 \%$, respectively ( $p=0.636$ ) (Figure $2 \mathrm{~A})$, and the 5-year DFS rate was $53.3 \%$ and $46.3 \%(p=0.377)$ (Figure $2 \mathrm{~B})$, respectively.

Correlations of GPX2 expression with the depth of invasion. Next, we investigated the GPX2 expression status for most invasive area in tumor lesions. Table II summarizes the intensity of GPX2 expression according to each pT stage; however, there was no statistically significant difference between the stages $(p=0.456)$.

We then investigated GPX2 expression in the specimens that contained pTa, pT1, and pT2 lesions of the same tumor in 17 patients. GPX normal and weak staining was observed in $47.1 \%$ and $52.9 \%$, of the lesions, respectively in $\mathrm{Ta}$ tumors. In contrast, GPX normal and weak staining was observed in $23.5 \%, 76.5 \%$ of the $\mathrm{T} 1$ lesions and $17.6 \%$ and
Table I. Relationship between GPX2 expression and clinicopathological characteristics.

\begin{tabular}{|c|c|c|c|}
\hline Parameter & $\begin{array}{l}\text { GPX2-normal } \\
\quad(n=26)\end{array}$ & $\begin{array}{l}\text { GPX2-weak } \\
\quad(\mathrm{n}=86)\end{array}$ & $p$-Value \\
\hline Age, median (range) & $69(54-82)$ & $68(34-85)$ & 0.169 \\
\hline Gender, n (\%) & & & 0.301 \\
\hline Male & $18(69.2)$ & $68(79.1)$ & \\
\hline Female & $8(30.8)$ & $18(20.9)$ & \\
\hline Pathologic tumor stage, n (\%) & & & 0.749 \\
\hline pT1 & $1(3.8)$ & $8(9.3)$ & \\
\hline pT2 & $12(46.2)$ & $41(47.7)$ & \\
\hline pT3 $\leq$ & $13(50.0)$ & $37(43.0)$ & \\
\hline Pathologic node stage, n (\%) & & & 1.00 \\
\hline Negative & $18(69.2)$ & $60(69.8)$ & \\
\hline Positive & $8(30.8)$ & $26(30.2)$ & \\
\hline Lymphovascular invasion, $\mathrm{n}(\%)$ & & & 0.357 \\
\hline Negative & $12(46.2)$ & $30(34.9)$ & \\
\hline Positive & $14(53.8)$ & $56(65.1)$ & \\
\hline Histology, n (\%) & & & 0.068 \\
\hline Urothelial carcinoma & $20(76.9)$ & $48(55.8)$ & \\
\hline Mixed variants & $6(23.1)$ & $38(44.2)$ & \\
\hline Neoadjuvant chemotherapy, n (\%) & & & 0.607 \\
\hline No & $21(80.8)$ & $64(74.4)$ & \\
\hline Yes & $5(19.2)$ & $22(25.6)$ & \\
\hline
\end{tabular}

GPX2: Glutathione peroxidase 2; pT: pathologic tumor.

$82.4 \%$ in T2 lesions, respectively. GPX2 expression levels of pT1 and T2 lesions were similar. The GPX2 expression levels showed significant decreases from pTa to pT1 compared to those from pT1 to pT2 (47.1\% vs. 5.9\%; $p=0.017$ ) (Figure 3A and B).

To assess the impact of GPX2 on tumor progression, we compared GPX2 expression for non-muscle invasive bladder cancer (NMIBC) in the same patients. Sixty patients (pTa: 30, pT1: 30) underwent TURBT before they received RC for recurrent MIBC. The pT1 tumors had a lower expression for GPX2 than that of pTa (63.3\% vs.93.3\%; $p=0.009)$ (Figure 4).

\section{Discussion}

In this study, immunohistochemical evaluations were performed to assess the GPX2 expression levels in patients with UC of the bladder. The IHC analysis showed a significant decrease in the levels of GPX2 expression $(76.8 \%)$ in those with UC. GPX2 expression was not associated with clinicopathologic characteristics or survival outcomes. However, GPX2 expression levels were significantly difference between those with Ta $v s$. T1 status in both MIBC and NMIBC cases.

Oxidative stress is generally considered to contribute to initiation and progression of carcinogenesis (16-18). Thus, an increase of GPX2 expression is believed have a preventive 

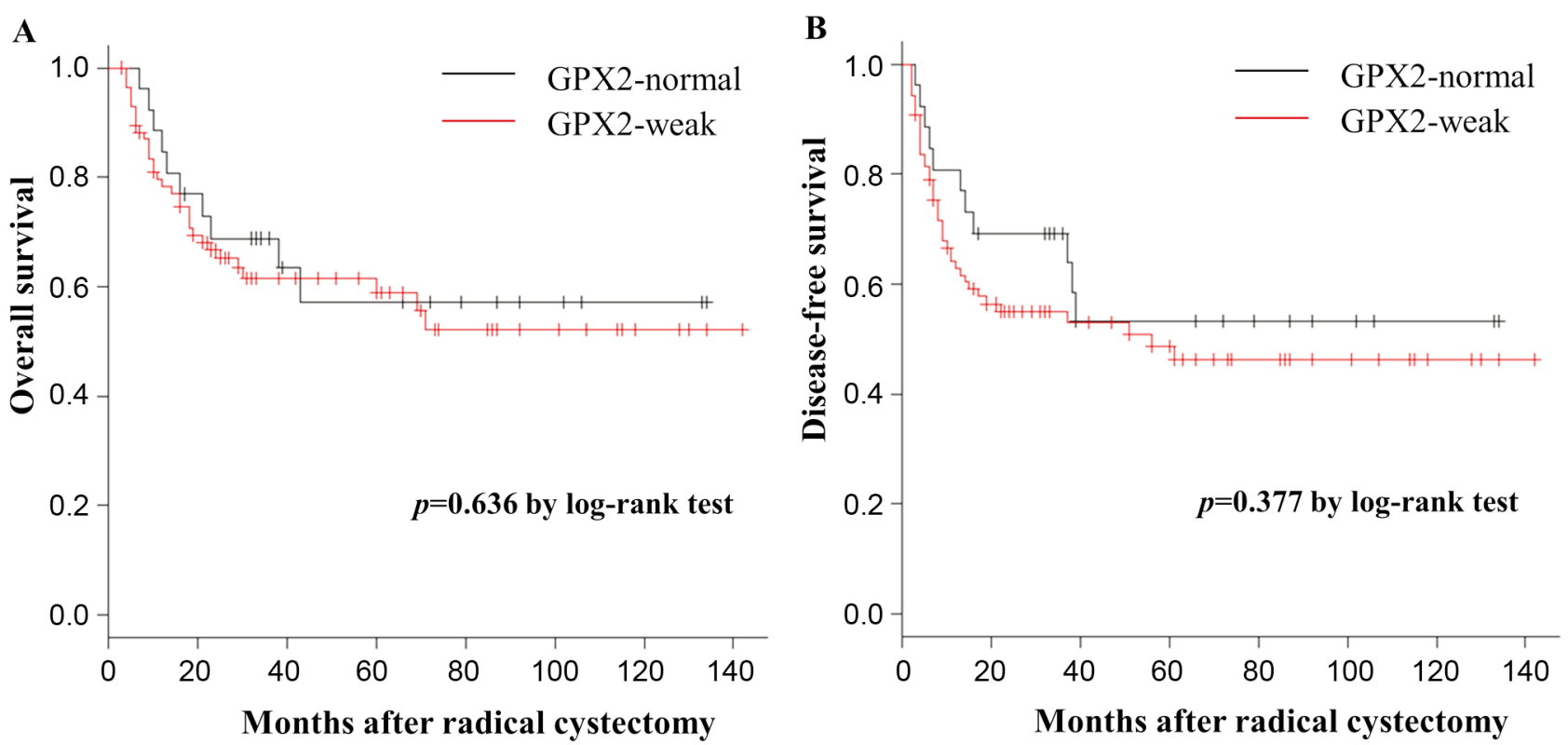

Figure 2. Kaplan-Meier curves show the prognostic analyses of GPX2 expression for overall survival (A) and disease-free survival (B) after radical cystectomy.

role for oxidative stress in epithelial cells (5). Two previous immunohistochemical studies have demonstrated relatively lower GPX2 expression rates of 49.1-51.6\% in those with bladder cancer $(19,20)$. In addition, low GPX2 expression levels were correlated with advanced stages of UC unlike those found in our results. These two previous studies also showed that GPX2-negative tumors had significantly higher risk of cancer-specific mortality. Although our IHC analyses for GPX2 expression have shown high negative rates in those with MIBC, the study has failed to reveal GPX2 expression value as a prognosticator. However, there was a clear difference observed in GPX2-staining intensity between the current and previous studies. In the present study, the GPX2staining intensity score was based on the proportion of cell positivity. In previous studies, scores were evaluated based on combining both the percentage and intensity of positive cells or an average intensity score.

In terms of survival analyses, Naiki et al. (14) reported that GPX2 overexpression was related to the prognosis of those with castration-resistant prostate cancer. Moreover, Lei et al. (8) demonstrated that low expression of GPX2 predicted poor prognosis in patients with esophageal squamous cell carcinoma. Regarding the relationship between antioxidant enzymes and bladder cancer, Arikan et al. (21) demonstrated that GPX activities were significantly lower in patients with UC when blood levels were examined. Brigelius-Flohe et al. (22) discussed how GPX2 knockdown cancer cells grew better in vitro and in vivo probably reflecting the physiological role of GPX2. However, the
Table II. Evaluation of GPX2 levels expression for most invasive areas according to pathologic tumor status in cystectomy specimens.

\begin{tabular}{lccc}
\hline GPX2 expression & pT1 $(\mathrm{n}=9)$ & $\mathrm{pT} 2(\mathrm{n}=53)$ & $\geq \mathrm{pT} 3(\mathrm{n}=50)$ \\
\hline $3+(\%)$ & $1(11.1)$ & $12(22.6)$ & $13(26.0)$ \\
$2+(\%)$ & $5(55.6)$ & $18(34.0)$ & $14(28.0)$ \\
$1+(\%)$ & $1(11.1)$ & $19(35.8)$ & $17(34.0)$ \\
$0(\%)$ & $2(22.2)$ & $4(7.5)$ & $6(12.0)$ \\
\hline
\end{tabular}

GPX2: Glutathione peroxidase 2; pT: pathologic tumor.

clear role of GPX2 in cancer progression and survival of patients with cancer remains undetermined; therefore, further investigations are required.

Interestingly, our study indicated significant decrease of GPX2 expression in T1 and T2 tumors compared to that in Ta tumors. This finding in early stages was not clearly observed among patients with UC in the previous two studies $(19,20)$. Banning et al. (23) reported that the role of GPX2 in carcinogenesis was studied in HT-29 clones, in which GPX2 expression was knocked down. GPX2 knockdown cells in HT-29 (a human colorectal adenocarcinoma cell line) had a higher capability to invade than the GPX2-expressing control cells (23). Therefore, the decreases in GPX2 expression could explain its potential to promote cancer invasion and progression from Ta to $\mathrm{T} 1$ status in those with UC. 


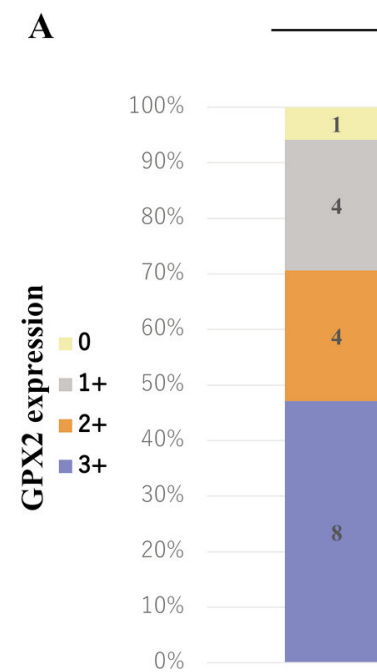

Ta
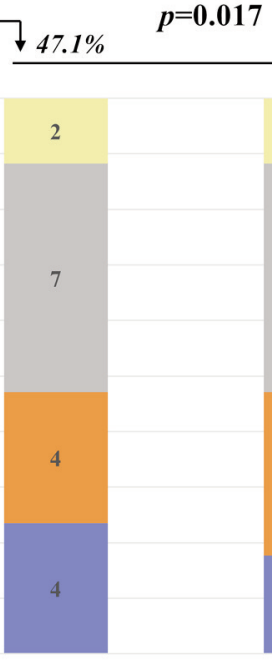

T1
B

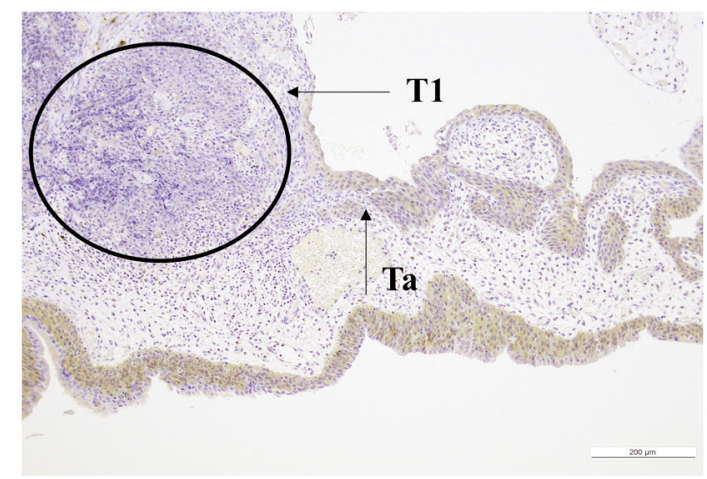

$\mathrm{n}=17$

Figure 3. (A) Comparison of GPX2 expression according to pT stage in cystectomy specimens. (B) GPX2 expression in pTa and pT1 lesions within the same tumor.

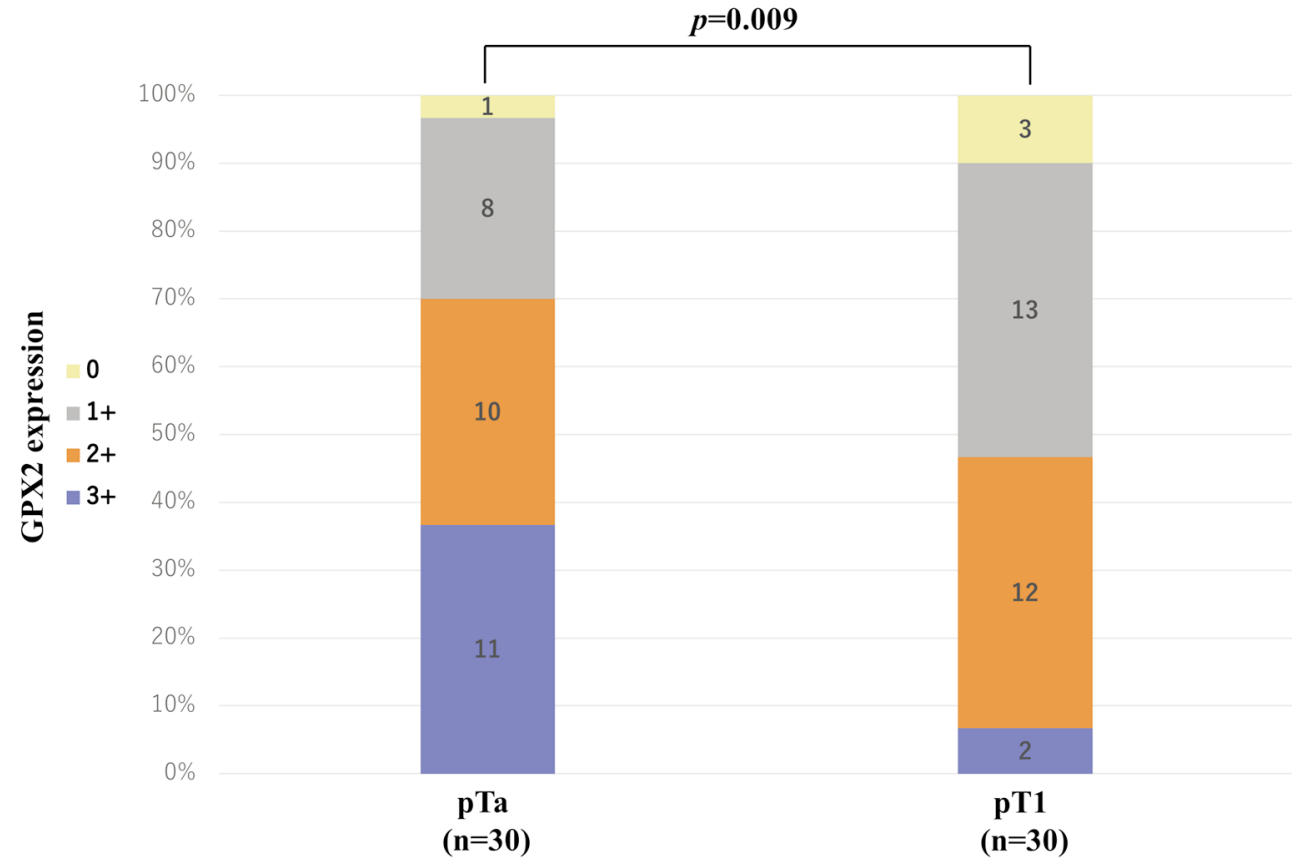

Figure 4. Evaluation of GPX2 expression in transurethral resection specimens at the time of non-muscle-invasive bladder cancer.

Morphological diversity of UC is common; however, UC with squamous differentiation (UCSD) is the most common variant that exists mainly in MIBC (24). Naiki et al. (20) reported that GPX2 positivity correlated with a significantly higher risk of mortality in those with UCSD unlike UC. However, we did not observe a significant difference in GPX2 expression among those with UCSD, compared to those with pure UC (data not shown). Further studies are 
required to better understand the association between GPX2 expression and variant type of UC.

There are certain limitations to this study such as its retrospective nature and small sample size. The treatment was not uniform, as some patients received $\mathrm{RC}$ alone while others received $\mathrm{RC}$ with neoadjuvant chemotherapy. For the immunohistochemical analysis, the study did not include a comparison of normal urothelial tissues in individuals without bladder cancer. However, despite these limitations, the strength of our cohort is that GPX2 expression levels showed significant decreases from pTa to pT1 in both MIBC and NMIBC cases. We believe that our study will assist in better understanding of tumor progression in UC. Further studies may help clarify the role of GPX2 expression in bladder cancer progression and help determine whether GPX2 is a target molecule for cancer invasion prevention.

\section{Conclusion}

The decrease in GPX2 expression in UC may be associated with early phases of tumor invasion in patients with UC of the bladder.

\section{Conflicts of Interest}

The Authors declare no potential conflicts of interest with respect to the research, authorship, and/or publication of this article.

\section{Authors' Contributions}

All Authors contributed to the design of the study, acquisition and interpretation of the data. All Authors discussed, verified and approved the final version of the article.

\section{References}

1 Miller KD, Siegel RL, Lin CC, Mariotto AB, Kramaer JL, Rowland JH, Stein KD, Alteri R and Jemal A: Cancer treatment and survivorship statistics, 2016. CA Cancer J Clin 66(4): 271289, 2016. PMID: 27253694. DOI: 10.3322/caac.21349

2 Bayraktar N, Kilic S, Bayraktar MR and Aksoy N: Lipid peroxidation and antioxidant enzyme activities in cancerous bladder tissue and their relation with bacterial infection: a controlled clinical study. J Clin Lab Anal 24(1): 25-30, 2010. PMID: 20087949. DOI: 10.1002/jcla.20356

3 Cao M, Mu X, Jiang C, Yang G, Chen H and Xue W: Singlenucleotide polymorphisms of GPX1 and MnSOD and susceptibility to bladder cancer: a systematic review and metaanalysis. Tumour Biol 35(1): 759-764, 2014. PMID: 24037914. DOI: $10.1007 / \mathrm{s} 13277-013-1103-6$

4 Ishaq M, Khan MA, Sharma K, Sharma G, Dutta RK and Majumdar S: Gambogic acid induced oxidative stress dependent caspase activation regulates both apoptosis and autophagy by targeting various key molecules (NF-kappaB, Beclin-1, p62 and NBR1) in human bladder cancer cells. Biochim Biophys Acta 1840(12): 3374-3384, 2014. PMID: 25218692. DOI: 10.1016/ j.bbagen.2014.08.019
5 Brigelius-Flohe R and Kipp AP: Physiological functions of GPx2 and its role in inflammation-triggered carcinogenesis. Ann NY Acad Sci 1259: 19-25, 2012. PMID: 22758632. DOI: 10.1111/j.1749-6632.2012.06574.x

6 Brigelius-Flohe R and Maiorino M: Glutathione peroxidases. Biochim Biophys Acta 1830(5): 3289-3303, 2013. PMID: 23201771. DOI: 10.1016/j.bbagen .2012.11.020

7 Wingler K, Muller C, Schmehl K, Florian S and BrigeliusFlohe R: Gastrointestinal glutathione peroxidase prevents transport of lipid hydroperoxides in $\mathrm{CaCo}-2$ cells. Gastroenterology 119(2): 420-430, 2000. PMID: 10930377. DOI: $10.1053 /$ gast.2000.9521

8 Lei Z, Tian D, Zhang C, Zhao S and Su M: Clinicopathological and prognostic significance of GPX2 protein expression in esophageal squamous cell carcinoma. BMC Cancer 16: 410, 2016. PMID: 27388201. DOI: 10.1186/s12885-016-2462-3

9 Woenckhaus M, Klein-Hitpass L, Grepmeier U, Merk J, Pfeifer M, Wild P, Bettstetter M, Wuensch P, Blaszyk H, Hartmann A, Hofstaedter F and Dietmaier W: Smoking and cancer-related gene expression in bronchial epithelium and non-small-cell lung cancers. J Pathol 210(2): 192-204, 2006. PMID: 16915569. DOI: 10.1002/path.2039

10 Naiki-Ito A, Asamoto M, Hokaiwado N, Takahashi S, Yamashita $\mathrm{H}$, Tsuda H, Ogawa $\mathrm{K}$ and Shirai T: Gpx2 is an overexpressed gene in rat breast cancers induced by three different chemical carcinogens. Cancer Res 67(23): 11353-11358, 2007. PMID: 18056462. DOI: 10.1158/0008-5472.CAN-07-2226

11 Murawaki Y, Tsuchiya H, Kanbe T, Harada K, Yashima K, Nozaka K, Tanida O, Kohno M, Mukoyama T, Nishimuki E, Kojo H, Matura T, Takahashi K, Osaki M, Ito H, Yodoi J, Murawaki Y and Shiota G: Aberrant expression of selenoproteins in the progression of colorectal cancer. Cancer Lett 259(2): 218-230, 2008. PMID: 18054426. DOI: 10.1016/j.canlet.2007.10.019

$12 \mathrm{Liu} \mathrm{C}$, He $\mathrm{X}$, Wu $\mathrm{X}$, Wang $\mathrm{Z}$, Zuo $\mathrm{W}$ and $\mathrm{Hu} \mathrm{G}$ : Clinicopathological and prognostic significance of GPx2 protein expression in nasopharyngeal carcinoma. Cancer Biomark 19(3): 335-340, 2017. PMID: 28453466. DOI: 10.3233/CBM-160542

13 Liu T, Kan XF, Ma C, Chen LL, Cheng TT, Zou ZW, Li Y, Cao FJ, Zhang WJ, Yao J and Li PD: GPX2 overexpression indicates poor prognosis in patients with hepatocellular carcinoma. Tumour Biol 39(6): 1010428317700410, 2017. PMID: 2863 5398. DOI: $10.1177 / 1010428317700410$

14 Naiki T, Naiki-Ito A, Asamoto M, Kawai N, Tozawa K, Etani T, Sato S, Suzuki S, Shirai T, Kohri K and Takahashi S: GPX2 overexpression is involved in cell proliferation and prognosis of castration-resistant prostate cancer. Carcinogenesis 35(9): 19621967, 2014. PMID: 24562575. DOI: 10.1093/carcin/bgu048

15 Montironi R, Cheng L, Scarpelli M and Lopez-Beltran A: Pathology and genetics: Tumours of the urinary system and male genital system: Clinical implications of the $4^{\text {th }}$ edition of the WHO classification and beyond. Eur Urol 70(1): 120-123, 2016. PMID: 26996660. DOI: 10.1016/j.eururo.2016.03.011

16 Kawanishi S, Ohnishi S, Ma N, Hiraku Y, Oikawa S and Murata $\mathrm{M}$ : Nitrative and oxidative DNA damage in infection-related carcinogenesis in relation to cancer stem cells. Genes Environ 38: 26, 2017. PMID: 28050219. DOI: 10.1186/s41021-016-0055-7

17 Qiu M, Chen L, Tan G, Ke L, Zhang S, Chen H and Liu J: A reactive oxygen species activation mechanism contributes to JS$\mathrm{K}$-induced apoptosis in human bladder cancer cells. Sci Rep 5: 15104, 2015. PMID: 26458509. DOI: 10.1038/srep15104. 
18 Liu Y, Lan L, Huang K, Wang R, Xu C, Shi Y, Wu X, Wu Z, Zhang J, Chen L, Wang L, Yu X, Zhu H and Lu B: Inhibition of Lon blocks cell proliferation, enhances chemosensitivity by promoting apoptosis and decreases cellular bioenergetics of bladder cancer: potential roles of Lon as a prognostic marker and therapeutic target in baldder cancer. Oncotarget 5: 11209-11224, 2014. PMID: 25526030. DOI: 10.18632/oncotarget.2026

19 Chang IW, Lin VC, Hung CH, Wang HP, Lin YY, Wu WJ, Huang $\mathrm{CN}, \mathrm{Li} \mathrm{CC}, \mathrm{Li} \mathrm{WM}, \mathrm{Wu}$ JY and Li CF: GPX2 underexpression indicates poor prognosis in patients with urothelial carcinomas of the upper urinary tract and urinary bladder. World J Urol 33(11): 1777-1789, 2015. PMID: 25813210. DOI: $10.1007 / \mathrm{s} 00345-015-1522-7$

20 Naiki T, Naiki-Ito A, Iida K, Etani T, Kato H, Suzuki S, Yamashita Y, Kawai N, Yasui $\mathrm{T}$ and Takahashi S: GPX2 promotes development of bladder cancer with squamous cell differentiation through the control of apoptosis. Oncotarget 9(22): 15847-15859, 2018. PMID: 29662611. DOI: 10.18632/oncotarget.24627

21 Arikan S, Akcay T, Konukoglu D, Obek C and Kural AR: The relationship between antioxidant enzymes and bladder cancer. Neoplasma 52(4): 314-317, 2005. PMID: 16059648.
22 Brigelius-Flohe R and Kipp A: Glutathione peroxidases in different stages of carcinogenesis. Biochim Biophys Acta 1790(11): 15551568, 2009. PMID: 19289149. DOI: 10.1016/j.bbagen.2009.03.006

23 Banning A, Kipp A, Schmitmeier S, Löwinger M, Florian S, Krehl S, Thalmann S, Thierbach R, Steinberg P and Brigelius-Flohé R: Glutathione peroxidase 2 inhibits cyclooxygenase-2-mediated migration and invasion of HT-29 adenocarcinoma cells but supports their growth as tumors in nude mice. Cancer Res 68(23): 9746-9753, 2008. PMID: 19047153. DOI: 10.1158/0008-5472. CAN-08-1321

24 Gellert LL, Warrick J and Al-Ahmadie HA: Urothelial carcinoma with squamous differentiation - the pathologists' perspective. Urol Oncol 33(10): 437-443, 2015. PMID: 26321057. DOI: 10.1016/ j.urolonc.2015.07.018

Received December 26, 2020

Revised January 16, 2021

Accepted January 25, 2021 